\title{
Predicting microRNA precursors with a generalized Gaussian components based density estimation algorithm Chih-Hung Hsieh ${ }^{1}$, Darby Tien-Hao Chang ${ }^{* 2}$, Cheng-Hao Hsueh ${ }^{2}$, Chi-Yeh $\mathrm{Wu}^{2}$ and Yen-Jen Oyang ${ }^{1,3,4}$
}

Addresses: ${ }^{1}$ Department of Computer Science and Information Engineering, National Taiwan University, Taipei 106, Taiwan, ${ }^{2}$ Department of Electrical Engineering, National Cheng Kung University, Tainan, 70101, Taiwan, ${ }^{3}$ Institute of Networking and Multimedia, National Taiwan University, Taipei 106, Taiwan and ${ }^{4}$ Center for Systems Biology and Bioinformatics, National Taiwan University, Taipei 106, Taiwan

E-mail: Chih-Hung Hsieh - hsiehch@gmail.com; Darby Tien-Hao Chang* - darby@ee.ncku.edu.tw;

Cheng-Hao Hsueh -n2697212@mail.ncku.edu.tw; Chi-YehWu-n26972138@mail.ncku.edu.tw; Yen-Jen Oyang - yjoyang@csie.ntu.edu.tw

${ }^{*}$ Corresponding author

from The Eighth Asia Pacific Bioinformatics Conference (APBC 2010)

Bangalore, India $|8-2|$ January 2010

Published: 18 January 2010

BMC Bioinformatics 2010, I I(SuppI I):S52 doi: 10.1 I86/I47I-2105-II-SI-S52

This article is available from: http://www.biomedcentral.com/I47I-2 I05/II/SI/S52

(c) 2010 Hsieh et al; licensee BioMed Central Ltd.

This is an open access article distributed under the terms of the Creative Commons Attribution License (http://creativecommons.org/licenses/by/2.0), which permits unrestricted use, distribution, and reproduction in any medium, provided the original work is properly cited.

\begin{abstract}
Background: MicroRNAs (miRNAs) are short non-coding RNA molecules, which play an important role in post-transcriptional regulation of gene expression. There have been many efforts to discover miRNA precursors (pre-miRNAs) over the years. Recently, ab initio approaches have attracted more attention because they do not depend on homology information and provide broader applications than comparative approaches. Kernel based classifiers such as support vector machine (SVM) are extensively adopted in these ab initio approaches due to the prediction performance they achieved. On the other hand, logic based classifiers such as decision tree, of which the constructed model is interpretable, have attracted less attention.

Results: This article reports the design of a predictor of pre-miRNAs with a novel kernel based classifier named the generalized Gaussian density estimator $\left(G^{2} D E\right)$ based classifier. The $G^{2} D E$ is a kernel based algorithm designed to provide interpretability by utilizing a few but representative kernels for constructing the classification model. The performance of the proposed predictor has been evaluated with 692 human pre-miRNAs and has been compared with two kernel based and two logic based classifiers. The experimental results show that the proposed predictor is capable of achieving prediction performance comparable to those delivered by the prevailing kernel based classification algorithms, while providing the user with an overall picture of the distribution of the data set.
\end{abstract}

Conclusion: Software predictors that identify pre-miRNAs in genomic sequences have been exploited by biologists to facilitate molecular biology research in recent years. The $G^{2} D E$ employed 
in this study can deliver prediction accuracy comparable with the state-of-the-art kernel based machine learning algorithms. Furthermore, biologists can obtain valuable insights about the different characteristics of the sequences of pre-miRNAs with the models generated by the $G^{2} D E$ based predictor.

\section{Background}

MicroRNAs (miRNAs) are short RNAs ( 20-22 nt) that direct post-transcriptional regulation of target genes by arresting the translation of mRNAs or by inducing their cleavage [1]. Since the initial discovery of miRNAs in Caenorhabditis elegans, RNA molecules are regarded as not only a carrier of gene information, but also a mediator of gene expression [2,3]. Currently, 9539 experimentally verified miRNAs have been collected in the miRBase database [4].

Experimental miRNA identification is accomplished by directional cloning of endogenous small RNAs [5]. Considering both the time and cost of experimental methods, many computational approaches have been proposed [6]. The mature miRNAs is cleaved from a 70$120 \mathrm{nt}$ precursor (pre-miRNA) with a stable hairpin structure. Identifying this specific structure has became an important step in analyzing miRNAs [1]. The earliest computational approaches for discovering pre-miRNAs are mainly based on comparative techniques and can only discover pre-miRNAs that are closely homologous to known miRNAs [7-10]. Alternatively, scientists have resorted to $a b$ initio approaches to discover pre-miRNAs based on the characteristics of their secondary structures [11-15]. The ab initio approaches based predictors are more generally applicable than those that are based on homology searches, since the ab initio approaches do not rely on the existence of homologues. As a result, design of the $a b$ initio approaches based predictors has attracted more attention in recent years.

The basis of the ab initio approaches is to design a coding scheme that maps the sequence and structure characteristics of pre-miRNAs into distinguishable patterns of feature vectors. Then, a supervised learning algorithm, also commonly referred to as data classification, is invoked to discover pre-miRNAs in the query RNA sequence based on the associated feature vectors. In recent years, the design of the coding scheme for characterizing pre-miRNAs has been extensively studied and several different schemes, including base pairing propensity [16], folding energy [17], base pair distance [18], and degree of compactness [19], have been proposed. On the other hand, most people working on this subject have employed the existing kernel based data classification algorithms such as the hidden Markov model (HMM) $[20,21]$, the support vector machine
(SVM) [22,23], and the kernel density estimator [15] to build the predictors due to the superior prediction performance delivered by these algorithms [24]. Nevertheless, conventional logic based data classification algorithms such as decision trees $[25,26]$ and decision rules $[27,28]$ continue to play a major role in some applications due to the interpretability of the logic rules identified by these algorithms. Such a summarized view of the characteristics and distribution of the data set further provides valuable insights about the relations among different features and is highly desirable for indepth analysis of pre-miRNAs.

Aiming to provide the desirable functionalities of both the kernel based and the logic based data classification algorithms, the study presented in this article has exploited the generalized Gaussian density estimator $\left(G^{2} D E\right)$ that we have recently proposed [29]. The $\mathrm{G}^{2} \mathrm{DE}$ identifies a small number of generalized Gaussian components to model the distribution of the data set in the vector space. As a result, the user can examine the parameter values associated with these of Gaussian components to obtain an overview picture of the distribution of the data set. Furthermore, through in-depth analysis of the parameter values, the user can obtain valuable insights about the relations among different features.

\section{Results and discussion}

This section first describes the overall scheme of using $\mathrm{G}^{2} \mathrm{DE}$ to analyze pre-miRNAs. Each step of the analysis procedure is further elaborated in the Methods section. Next, the prediction performance of the employed classification algorithm is evaluated and compared with four classification algorithms. A demonstrative analysis is also presented to investigate the interpretability of the employed classification algorithm.

\section{Using $G^{2} D E$ to analyze pre-miRNAs}

This work uses only sequence information to identify pre-miRNAs from pseudo hairpins, which are RNA sequences with similar stem-loop features to premiRNAs but containing no mature miRNAs. In this method, each RNA sequence is represented as a feature vector. The characteristics used to generate the feature vector, including sequence composition, folding energy and stem-loop shape, have been shown to be useful for predicting pre-miRNAs in previous studies $[17,18,30]$. 
The main task carried out during the learning process of this method is to construct two mixture models of generalized Gaussian components for summarizing premiRNAs and pseudo hairpins in the vector space. We model this learning process as a large-parameter-optimization problem (LPOP) and employ an efficient optimization algorithm, Ranking-based Adaptive Mutation Evolutionary (RAME) [31], to decide the parameters associated with each generalized Gaussian component. Finally, the models learnt through the LPOP process are used to predict whether a query RNA sequence is a premiRNA. Furthermore, the constructed model of $\mathrm{G}^{2} \mathrm{DE}$ comprises a small number of generalized Gaussian components and is capable of detecting the sub-clusters or sub-classes of the data set. This study utilizes this feature of $\mathrm{G}^{2} \mathrm{DE}$ to develop a two-stage analysis where the first stage uses $G^{2} D E$ to partition the data set while the second stage uses $\mathrm{G}^{2} \mathrm{DE}$ to investigate each of the partitioned subsets.

\section{Prediction performance}

The present approach is evaluated using two datasets, HU920 and HU424, combined with four feature sets. See the Methods section for details of the datasets and the feature sets. The prediction performance is compared to two kernel based classifiers, SVM and RVKDE, and two logic based classifiers, C4.5 and RIPPER. The parameters for each classifier are determined by maximizing the prediction accuracy of ten-fold cross-validation on the HU920. A prediction is performed by using the HU920 dataset to predict the HU464 dataset with the selected parameters.

The employed $\mathrm{G}^{2} \mathrm{DE}$ classifier is compared with two kernel based classifiers, SVM and the relaxed variable kernel density estimator (RVKDE) [32]. The SVM is a commonly used classifier because of its prevailing success in diverse bioinformatics problems [14,33,34], while the RVKDE has been shown to have advantages for predicting species-specific pre-miRNAs [15]. Two well- known logic based classifiers, C4.5 [35] and RIPPER [36], are also included as representatives of logic based classification algorithms.

As shown in Table 1, the prediction accuracies of one-stage $\mathrm{G}^{2} \mathrm{DE}$ are $80.39 \%, 92.03 \%, 91.60 \%$ and $78.66 \%$ with different feature sets. The two-stage $\mathrm{G}^{2} \mathrm{DE}$ further improve the prediction accuracies and achieves the best average accuracy of $86.58 \%$. Though the number of kernels increases from one-stage to two-stage $\mathrm{G}^{2} \mathrm{DE}$, it is still much less than the other kernel based classifiers. Table 1 also reveals that the kernel based classifiers generally outperform the logic based classifiers. As a result, the $\mathrm{G}^{2} \mathrm{DE}$ is capable of delivering satisfactory performance using a smaller number of kernel functions than the other systems.

In addition to compare the alternative classification algorithms, the prediction performance of the proposed method is also compared to two existing pre-miRNA identification packages, miPred [14] and miR-KDE [15]. A number $(n f)$ of features from the four feature sets are selected with Wilcoxon rank sum test [37] are utilized as the feature set of $\mathrm{G}^{2} \mathrm{DE}$. In current implementation, $n f$ is set to seven because that the feature set yielding the best performance in Table 1 contains seven features. In this experiment, a prediction is performed by using the HU920 dataset to predict the HU464 dataset with the parameters maximizing the prediction accuracy of tenfold cross-validation on the HU920 dataset. The five indices for binary classification (Table 2) used in miPred and miR-KDE are adopted. Table 3 shows the experimental results. $\mathrm{G}^{2} \mathrm{DE}$ achieves comparable performance with those delivered by miPred and miR-KDE. A notable difference between $\mathrm{G}^{2} \mathrm{DE}$ and miPred and miR-KDE is that $\mathrm{G}^{2} \mathrm{DE}$ utilizes much less kernels. $\mathrm{G}^{2} \mathrm{DE}-2$ yields the best \%ACC, \%SE, \%Fm and \%MCC, which are 93.32\%, $90.09 \%, 93.10 \%$ and $87.16 \%$, respectively. Although the number of kernels in $\mathrm{G}^{2} \mathrm{DE}-2$ is five times larger than that in $\mathrm{G}^{2} \mathrm{DE}$, it is more acceptable to perform further analyses than that in miPred and miR-KDE.

Table I: Prediction accuracies achieved by SVM, RVKDE, G²DE, C4.5 and RIPPER

\begin{tabular}{|c|c|c|c|c|c|c|}
\hline \multirow[b]{2}{*}{ Feature set } & \multicolumn{4}{|c|}{ Kernel based classifiers } & \multicolumn{2}{|c|}{ Logic based classifiers } \\
\hline & SVM & RVKDE & $G^{2} D E$ & $G^{2} D E-2$ & C4.5 & RIPPER \\
\hline I & $80.17 \%$ & $77.59 \%$ & $80.39 \%$ & $80.60 \%$ & $77.80 \%$ & $76.72 \%$ \\
\hline 2 & $93.32 \%$ & $92.46 \%$ & $92.03 \%$ & $93.10 \%$ & $90.95 \%$ & $90.52 \%$ \\
\hline 3 & $91.60 \%$ & $91.16 \%$ & $91.60 \%$ & $92.46 \%$ & $91.16 \%$ & $91.38 \%$ \\
\hline 4 & $78.66 \%$ & $79.53 \%$ & $78.66 \%$ & $80.17 \%$ & $77.37 \%$ & $76.72 \%$ \\
\hline Average & $85.94 \%$ & $85.18 \%$ & $85.67 \%$ & $86.58 \%$ & $84.32 \%$ & $83.84 \%$ \\
\hline \#kernels & 361 & 920 & 6 & 36 & 10 & 9 \\
\hline
\end{tabular}

The best performance among each feature set is highlighted with bold font. The $G^{2} D E-2$ indicates the two-stage $G^{2} D E$, which uses the first stage $G^{2} D E$ to cluster samples and than uses the second stage $G^{2} D E$ to classify each clusters. The \#kernels indicate number of kernels in average, where the numbers of logic based classifiers indicate the number of rules they deliver. 
Table 2: Evaluation measures employed in this study

\begin{tabular}{lll}
\hline Measure & Abbreviation & Equation \\
\hline Sensitivity (recall) & $\% S E$ & TP/(TP+FN) \\
Specificity & $\% S P$ & TN $/(T N+F P)$ \\
Accuracy & $\% A C C$ & $(T P+T N) /(T P+T N+F P+F N)$ \\
F-measure & $\% F m$ & $2 T P /(2 T P+F P+F N)$ \\
Matthews' correlation coefficient & $\% M C C$ & $(T P \times T N-F P \times F N) / s q r t((T P+F P) \times(T N+F N) \times(T P+F N) \times(T N+F P))$ \\
\hline
\end{tabular}

The definition of the abbreviations used: TP is the number of real pre-miRNAs detected; FN is the number of real pre-miRNAs missed; TN is the number of pseudo hairpins correctly classified; and FN is the number of pseudo hairpins incorrectly classified as pre-miRNA.

Table 3: Comparison of $G^{2} D E$ and two existing pre-miRNA identification packages

\begin{tabular}{lllllll}
\hline Method & \#kernels & \%SE & \%SP & \%ACC & \%Fm & \%MCC \\
\hline miPred & 280 & $88.80 \%$ & $96.55 \%$ & $92.67 \%$ & $92.38 \%$ & $85.60 \%$ \\
miR-KDE & 920 & $89.22 \%$ & $96.12 \%$ & $92.67 \%$ & $92.41 \%$ & $85.55 \%$ \\
G 2 DE & 6 & $87.07 \%$ & $97.84 \%$ & $92.46 \%$ & $92.03 \%$ & $85.41 \%$ \\
G $^{2}$ DE-2 & 36 & $\mathbf{9 0 . 0 9 \%}$ & $96.55 \%$ & $\mathbf{9 3 . 3 2 \%}$ & $\mathbf{9 3 . 1 0} \%$ & $\mathbf{8 7 . 1 6 \%}$ \\
\hline
\end{tabular}

The best performance among each evaluation index is highlighted with bold font. The $G^{2} D E-2$ indicates the two-stage $G^{2} D E$, which uses the first stage $G^{2} D E$ to cluster samples and than uses the second stage $G^{2} D E$ to classify each clusters.

\section{Interpretability of $\mathbf{G}^{2} \mathrm{DE}$}

Though the two-stage $\mathrm{G}^{2} \mathrm{DE}$ achieves the best performance in Table 1, the small differences to other classifiers suggests that pre-miRNA prediction algorithms have reached the maximum with current feature sets. Hence, how to interpret the learnt model of machine learning techniques for users is crucial in pre-miRNA prediction. In this subsection, the second feature set is used as an example to explain how to interpret the models generated by the $\mathrm{G}^{2} \mathrm{DE}$ based predictor. Figure 1 shows the parameters associated with the three Gaussian components used to summarize the pre-miRNAs in the HU920 dataset. To analyze these parameters, we compare them to the Pearson product-moment correlation coefficients (PMCC) [38] of the pre-miRNAs in the HU920 dataset (Figure 2). Parameters in the models generated by $\mathrm{G}^{2} \mathrm{DE}$ that differ more from the corresponding elements obtained by PMCC are more of our interest. For instance, in Figure 2, the correlation between the first feature $(m f e 2)$ and the fifth feature (dQ) of this feature set is 0.36. See the 'Feature set' subsection for detailed explanations of these features. On the other hand, the correlations between the two features in the three Gaussian components (shown in Figure 1) are $0.12,0.08$ and 0.02 , all of which are relatively lower than 0.36 . As PMCC summarizes the distribution of all pre-miRNAs, this analysis suggests that the HU920 dataset is composed of multiple clusters of samples, where the relation between $m f e 2$ and $d Q$ varies in different clusters and causes the inconsistency of correlations.

To verify the above analysis, this study depicts the HU920 dataset with their $m f e 2$ and $d Q$ values (Figure 3).
In Figure 3, the red squares and green circles represent the pre-miRNAs and the pseudo hairpins, respectively. The red ellipses, named $G G C_{1}, G G C_{2}$ and $G G C_{3}$, are the generalized Gaussian components shown in Figure 1, and the black ellipse is the Gaussian distribution shown in Figure 2. Figure 3 reveals that there are potentially two clusters of pre-miRNAs in this dataset and form a shape of a mirrored ' $\mathrm{L}$ ' in the feature space of $m f e 2$ and $d Q$. $m f e 2$ measures the energy of folding while $d Q$ measures the arrangement of base paring. Table 4 shows the detailed descriptions of these features. Figure 3 suggests that if a RNA sequence has low folding energy, it is probably a pre-miRNA regardless of the arrangement of its base paring. On the other hand, there is another cluster of pre-miRNAs that have similar folding energies to those of pseudo hairpins. No obvious correlation exists in both clusters of pre-miRNAs. These findings coincide with the analysis based on the models generated by $\mathrm{G}^{2} \mathrm{DE}$. In this example, the Gaussian components learnt by $\mathrm{G}^{2} \mathrm{DE}$ help users to identify features of interest without plotting all pairs of features along with the relations between them.

Another useful analysis provided by the learnt model of the $\mathrm{G}^{2} \mathrm{DE}$ based predictor is sub-class detection. By defining that a sample belongs to the Gaussian component reporting the maximum function value at the location of that sample, the learnt Gaussian components of $\mathrm{G}^{2} \mathrm{DE}$ suggests that "a sample that belongs to $G G C_{2}$ is a pre-miRNA." This statement is similar to the normal decision rule "a sample that has $m f e 2<0.4$ is a premiRNA," except that the conditions (belong vs. mfe $2<$ 0.4 ) within the rule inferred by $\mathrm{G}^{2} \mathrm{DE}$ is non-linear. This non-linearity of a single rule is an important feature of 
$\mathrm{G}^{2} \mathrm{DE}$ to describe more complicated model than traditional logic based classifiers when the number of kernels of $G^{2} \mathrm{DE}$ is limited to the number of rules of logic based algorithms. One immediate application of the sub-class detection is to group samples into clusters and then construct a classifier for each of the clusters. The performance improved by applying such two-stage framework has been shown in the previous subsection.

Generalized Gaussian component $1\left(G G C_{1}\right)$ :

Mean $=[0.58,0.27,0.82,0.18,-0.55,-0.93,-0.37]$;

Std. $=[0.78,0.63,0.84,0.67,0.60,0.43,0.81]$

Corr. matrix $=\left[\begin{array}{ccccccc}1.00 & 0.15 & -0.66 & 0.56 & 0.12 & 0.05 & 0.41 \\ 0.15 & 1.00 & 0.52 & -0.43 & -0.06 & -0.80 & 0.44 \\ -0.66 & 0.52 & 1.00 & -0.53 & -0.17 & -0.56 & 0.03 \\ 0.56 & -0.43 & -0.53 & 1.00 & 0.00 & 0.42 & 0.42 \\ 0.12 & -0.06 & -0.17 & 0.00 & 1.00 & -0.04 & -0.07 \\ 0.05 & -0.80 & -0.56 & 0.42 & -0.04 & 1.00 & -0.29 \\ 0.41 & 0.44 & 0.03 & 0.42 & -0.07 & -0.29 & 1.00\end{array}\right]$.

Weight : 0.826

Generalized Gaussian component $2\left(G G C_{2}\right)$ :

Mean $=[0.09,-0.21,0.73,-0.19,0.09,-0.70,0.28]$;

Std. $=[0.14,0.79,0.47,0.19,0.76,0.81,0.50]$;

Corr. matrix $=\left[\begin{array}{ccccccc}1.00 & 0.04 & -0.43 & -0.12 & 0.08 & -0.34 & 0.42 \\ 0.04 & 1.00 & -0.01 & 0.22 & -0.42 & -0.03 & -0.33 \\ -0.43 & -0.01 & 1.00 & 0.14 & 0.55 & -0.09 & 0.28 \\ -0.12 & 0.22 & 0.14 & 1.00 & 0.33 & 0.29 & -0.10 \\ 0.08 & -0.42 & 0.55 & 0.33 & 1.00 & -0.09 & 0.71 \\ -0.34 & -0.03 & -0.09 & 0.29 & -0.09 & 1.00 & -0.69 \\ 0.42 & -0.33 & 0.28 & -0.10 & 0.71 & -0.69 & 1.00\end{array}\right]$.

Weight : 0.488

Generalized Gaussian component $3\left(G G C_{3}\right)$ :

Mean $=[-0.04,-0.49,0.24,0.36,-0.40,0.62,0.73]$;

Std. $=[0.66,0.93,0.91,0.49,0.43,0.73,0.33]$;

Corr. matrix $=\left[\begin{array}{ccccccc}1.00 & 0.62 & -0.76 & 0.81 & 0.02 & -0.16 & -0.75 \\ 0.62 & 1.00 & -0.84 & 0.58 & -0.41 & -0.06 & -0.61 \\ -0.76 & -0.84 & 1.00 & -0.76 & 0.45 & 0.07 & 0.57 \\ 0.81 & 0.58 & -0.76 & 1.00 & -0.27 & 0.15 & -0.56 \\ 0.02 & -0.41 & 0.45 & -0.27 & 1.00 & -0.43 & -0.16 \\ -0.16 & -0.06 & 0.07 & 0.15 & -0.43 & 1.00 & 0.54 \\ -0.75 & -0.61 & 0.57 & -0.56 & -0.16 & 0.54 & 1.00\end{array}\right]$.

Weight : 0.545

\section{Figure I}

Parameters of the three generalized Gaussian components generated by $\mathbf{G}^{2} \mathbf{D E}$. This figure shows the three generalized Gaussian components of $G^{2} D E$ with the pre-miRNAs in the HU920 dataset and the second feature set. The correlation of interest is indicated with an arrow.
Mean $=[0.63,0.18,0.24,0.01,-0.71,-0.64,-0.61]$;

Std. $=[0.26,0.35,0.31,0.33,0.23,0.27,0.30]$

Corr. matrix $=\left[\begin{array}{ccccccc}1.00 & 0.63 & -0.42 & 0.67 & 0.36 & 0.37 & -0.90 \\ 0.63 & 1.00 & -0.62 & 0.47 & 0.55 & 0.54 & -0.56 \\ -0.42 & -0.62 & 1.00 & -0.59 & -0.48 & -0.45 & 0.20 \\ 0.67 & 0.47 & -0.59 & 1.00 & 0.38 & 0.37 & -0.32 \\ 0.36 & 0.55 & -0.48 & 0.38 & 1.00 & 0.99 & -0.28 \\ 0.37 & 0.54 & -0.45 & 0.37 & 0.99 & 1.00 & -0.30 \\ -0.90 & -0.56 & 0.20 & -0.32 & -0.28 & -0.30 & 1.00\end{array}\right]$.

\section{Figure 2}

Parameters obtained by basic statistics. These parameters are obtained by calculating the mean, standard deviation and Pearson product-moment correlation coefficients with the pre-miRNAs of the HU920 dataset and the second feature set. The correlation of interest is indicated with an arrow.

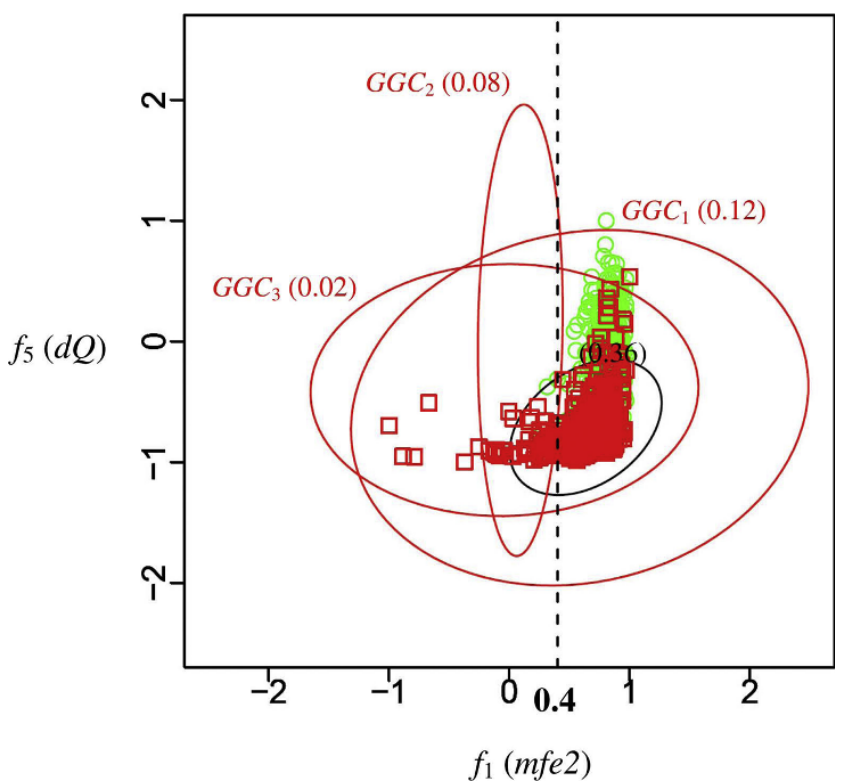

Figure 3

Distribution of the HU920 dataset. The $x$-axis is the first feature of the second feature set, ratio of MFE to the number of stems; the $y$-axis is the fifth feature of the second feature set, adjusted Shannon entropy. Red ellipses represent the generalized Gaussian components shown in Figure I; the black ellipse represents the Gaussian component shown in Figure 2. The red squares and green circles represent the pre-miRNAs and the pseudo hairpins, respectively. Values within the parentheses indicate the correlations between these two features in the corresponding Gaussian components. 
Table 4: Summary of the adopted feature sets

\begin{tabular}{ll}
\hline Feature & Description \\
\hline $\begin{array}{l}\text { Set I } \\
A A, A C, \ldots, U U \\
\% G+C\end{array}$ & $\begin{array}{l}\text { Frequencies of } 16 \text { dinucleotide pairs } \\
\text { Set } 2\end{array}$ \\
$m f e 2$ & Percentage of nitrogenous bases which are either $G$ or $C$ \\
$m f e I$ & Ratio of $d G$ to the number of stems \\
$d P$ & Ratio of $d G$ to $\% G+C$ \\
& Adjusted base pairing propensity. $d P$ is the number of base pairs observed in the secondary structure divided by the \\
$d G$ & sequence length. \\
$d Q$ & Adjusted minimum free energy of folding. $d G$ is the minimum free energy (MFE) divided by the sequence length. \\
$d D$ & Adjusted Shannon entropy. $d Q$ measures the entropy of the base pairing probability distribution (BPPD). \\
$d F$ & Adjusted base pair distance. $d D$ measures the average distance between all base pairs of structures inferred from the \\
Set 3 & sequence. \\
$z G, z Q, z D, z P, z F$ & Compactness of the tree-graph representation of the sequence. \\
Set 4 & 5 normalized variants of $d P, d G, d Q, d D$ and $d F$ \\
$I H$ & Hairpin length \\
$I L$ & Loop length \\
$I C$ & Consecutive base-pairs \\
$\% L$ & Ratio of loop length to hairpin length \\
\hline
\end{tabular}

The table shows the order of a feature within the feature set. For example, the fifth feature in the second feature set is $d Q$.

\section{Conclusion}

Software predictors that identify pre-miRNAs in genomic sequences have been exploited by biologists to facilitate molecular biology research in recent years. However, design of advanced predictors of pre-miRNAs has focused mostly on coding the distinguishable sequence as well as structure characteristics of miRNAs. The study presented in this article addresses this issue from the aspect of exploiting advanced machine learning algorithms. The $\mathrm{G}^{2} \mathrm{DE}$ employed in this study has been designed to deliver prediction accuracy comparable with the state-of-the-art kernel based machine learning algorithms, while providing the user with good interpretability. As demonstrated by the experiments reported in this study, the models generated by the $\mathrm{G}^{2} \mathrm{DE}$ based classifier provide the user with crucial clues about the different characteristics of the sequences of pre-miRNAs.

\section{Methods}

\section{Feature set}

This work adopts 33 characteristic features which have been shown to be useful for miRNA detection in previous studies [16-19,30,39-41]. To investigate how alternative classifiers perform when using different features, these features are grouped as four different sets according to their biochemical properties. The first feature set includes 17 sequence composition variables, which comprise frequencies of 16 dinucleotide pairs and proportion of $\mathrm{G}$ and $\mathrm{C}$ in the RNA molecule. The second feature set includes seven folding measures: Minimum Free Energy (MFE) and two of its variants [17,18,39], base pairing propensity [16], Shannon entropy [18], base pair distance $[18,40]$ and degree of compactness $[19,41]$. The third feature set uses the Z-score [42] to normalize the features in the second feature set except the two MFE variants. The fourth feature set includes four stem-loop features: hairpin length [15], loop length [15], consecutive base-pairs [15] and the ratio of loop length to hairpin length [15]. Table 4 shows a summary of these features.

\section{Dataset}

The process of data preparation is the same as that in the compared pre-miRNA identification packages $[14,15]$ for a fair comparison. 692 human miRNA precursors are collected from the miRBase registry database [43] (release 12.0) as the positive set. For the negative set, 8494 pseudo hairpins collected from the protein-coding regions (CDSs) according to RefSeq [44] and UCSC refGene [45] annotations are analyzed. These RNA sequences are extracted from genomic regions where no experimentally validated splicing event has been reported [12]. The secondary structures of the 8494 RNA sequences are obtained by executing RNAfold [46]. RNA sequences with $<18$ base pairs on the stem, MFE $>-25$ $\mathrm{kcal} / \mathrm{mol}$ and multiple loops are excluded. As a result, 3988 pseudo hairpins, which are similar to genuine premiRNAs in terms of length, stem-loop structure, and number of bulges but not have been reported as premiRNAs, are used as the negative set.

Based on the positive and negative sets, one training set and one testing set are built to evaluate the pre-miRNA predictors. The training set, HU920, comprises 460 
human pre-miRNAs and 460 pseudo hairpins randomly selected from the positive and negative sets, respectively. The HU920 dataset is used for parameter selection and model construction of the pre-miRNA predictors. The testing set, HU464, comprises the remaining 232 human pre-miRNAs and 232 randomly selected pseudo hairpins. Care has been taken to ensure that no pseudo hairpin is included in both datasets. Before performing any experiments on these datasets, the features are rescaled linearly by the svm-scale program [47] to the interval of $[-1.0,1.0]$.

\section{Generalized Gaussian density estimator}

This work transforms samples into feature vectors and then uses them to construct a generalized Gaussian density estimator $\left(G^{2} D E\right)$ [29]. A density estimator is in fact an approximate probability density function. With the $\mathrm{G}^{2} \mathrm{DE}$ algorithm, one approximate probability density function of the following form is generated for each class of samples:

$$
\begin{aligned}
& \hat{f}(\mathbf{v})=\frac{1}{\sum w_{i}} \sum \frac{1}{(2 \pi)^{d / 2}} \operatorname{GGC}\left(w_{i}, \boldsymbol{\mu}_{i}, \boldsymbol{\Sigma}_{i}\right) \text { and } \\
& \operatorname{GGC}\left(w_{i}, \boldsymbol{\mu}_{i}, \boldsymbol{\Sigma}_{i}\right)=w_{i} \frac{1}{\left|\Sigma_{i}\right|^{1 / 2}} \exp \left(-\frac{1}{2}\left(\mathbf{v}-\boldsymbol{\mu}_{i}\right)^{\mathrm{T}} \boldsymbol{\Sigma}_{i}^{-1}\left(\mathbf{v}-\boldsymbol{\mu}_{i}\right)\right),
\end{aligned}
$$

where $d$ is the dimension of the vector space and $w_{i,} \boldsymbol{\mu}_{i}$ and $\boldsymbol{\Sigma}_{i}$ are the weight, mean vector, and the covariance matrix of the $i$-th Gaussian component. Let $\hat{f}_{j}$ denote the approximate probability density function for the $j$-th class of samples. Then, a query sample located at $\mathbf{v}$ is predicted to belong to the class of which the corresponding likelihood function defined in the following gives the maximum value:

$$
L_{h}(\mathbf{v})=\frac{\left|S_{h}\right| \cdot \hat{f}_{h}(\mathbf{v})}{\sum_{j}\left|S_{j}\right| \cdot \hat{f}_{j}(\mathbf{v})},
$$

where $S_{j}$ is the set of class- $j$ training samples and $\left|S_{j}\right|$ is the number of class- $j$ training samples.

In current $\mathrm{G}^{2} \mathrm{DE}$ implementation, the user can specify the maximum number of generalized Gaussian components that can be incorporated to generate the approximate probability density function for one class of samples. If the user sets this number to $k$ and the total number of features of the data set is $d$, then the learning algorithm of $\mathrm{G}^{2} \mathrm{DE}$ needs to figure out the optimal combination of the values of the following $k(d+2)(d+1) / 2$ parameters in order to generate one approximate probability density function: $k d$ dimensional vectors as the means of the generalized Gaussian components; $k$ sets of $d(d+1) / 2$ coefficients with each corresponding to the covariance matrix of one generalized Gaussian components; $k$ weights. The optimal combination of parameter values are figured out using the Ranking-based Adaptive Mutation Evolutionary (RAME) algorithm [31].

In the evolutionary optimization algorithm, the objective function to be maximized is as follows:

$$
\mathrm{O}(\mathrm{Z})=\# \text { Correct }+\theta \cdot \sum_{j} \log (\text { likelihood of class } j)
$$

where

(1) $\mathrm{Z}$ is the vector formed by concatenating all the $k$ $(d+2)(d+1) / 2$ parameters associated with the approximate probability density function of one class of samples;

(2) \#Correct is the number of correctly classified training samples;

(3) $\theta$ is a user-defined parameter;

(4) likelihood of class $j=\prod_{\mathbf{s}_{i} \in \text { class } j} \hat{f}_{j}\left(\mathbf{s}_{i}\right)$, where $\mathbf{s}_{i}$ is the $i$-th training sample of class $j$.

The objective function adopted in the learning process of $\mathrm{G}^{2} \mathrm{DE}$ consists of two terms. Both terms have specific mathematical meanings. Maximizing term \#Correct implies that the number of training samples of which the class can be correctly predicted with the decision model is maximized. Meanwhile, maximizing the second term implies that the mixture models give the maximum likelihood with the training samples.

\section{Two-stage $G^{2} D E$}

The learnt model of $\mathrm{G}^{2} \mathrm{DE}$ is composed of a small number of Gaussian components. In this study, a sample is defined as belonging to the Gaussian component reporting the maximum function value at the location of that sample. The two-stage classification framework is performed by first grouping samples belonging to the same Gaussian component into clusters and then constructing a classifier for each of the clusters. In the first stage, all training samples would be submitted to $G^{2} D E$ for constructing a mixture model of generalized Gaussian components. Suppose that the learnt model contains $n_{1}$ generalized Gaussian components, essentially dividing the training dataset into $n_{1}$ clusters. Each training sample would then be assigned to the Gaussian component to which it belongs. In the second stage, $\mathrm{G}^{2} \mathrm{DE}$ is invoked $n_{1}$ times to construct $n_{1}$ mixture models for each cluster. If each of the learnt models in the second stage contains $n_{2}$ generalized Gaussian components, the final classifier will contain $n_{1} \times n_{2}$ generalized Gaussian components. 


\section{Competing interests}

The authors declare that they have no competing interests.

\section{Authors' contributions}

Author C.-H. Hsieh performed all calculations and analysis and drafted the manuscript. Author D. T.-H. Chang aided in design of the methodology, interpretation of the data and manuscript preparation. Author C.-H. Hsueh and C.-Y. Wu participated in the data preparation. Author Y.-J. Oyang conceived the design of $\mathrm{G}^{2} \mathrm{DE}$ classifier. All authors have read and approved this manuscript.

\section{Acknowledgements}

The authors would like to thank the National Science Council of the Republic of China, Taiwan, for financially supporting this research under Contract Nos NSC 97-2627-P-00I-002, NSC 96-2320-B-006-027-MY2 and NSC 96-222I-E-006-232-MY2.

This article has been published as part of BMC Bioinformatics Volume II Supplement 1, 2010: Selected articles from the Eighth Asia-Pacific Bioinformatics Conference (APBC 2010). The full contents of the supplement are available online at http://www.biomedcentral.com//47I$\underline{2105 / I 1 \text { ? issue }=\mathrm{SI}}$.

\section{References}

I. Bartel DP: MicroRNAs: Genomics, biogenesis, mechanism, and function. Cell 2004, I I6(2):28I-297.

2. Lee RC, Feinbaum RL and Ambros V: The C-Elegans Heterochronic Gene Lin-4 Encodes Small RNAs with Antisense Complementarity to Lin- I4. Cell 1993, 75(5):843-854.

3. Reinhart BJ, Slack FJ, Basson M, Pasquinelli AE, Bettinger JC, Rougvie AE, Horvitz HR and Ruvkun G: The 2I-nucleotide let-7 RNA regulates developmental timing in Caenorhabditis elegans. Nature 2000, 403(6772):90I-906.

4. Griffiths-Jones S, Saini HK, van Dongen S and Enright AJ: miRBase: tools for microRNA genomics. Nucleic Acids Res 2008, 36: DI54-DI58.

5. Chen PY, Manninga H, Slanchev K, Chien MC, Russo JJ, Ju JY, Sheridan R, John B, Marks DS and Gaidatzis D, et al: The developmental miRNA profiles of zebrafish as determined by small RNA cloning. Genes \& Development 2005, 19 (I I): 1288-1293.

6. Berezikov E, Cuppen E and Plasterk RHA: Approaches to microRNA discovery. Nature Genetics 2006, 38:S2-S7.

7. Boffelli D, McAuliffe J, Ovcharenko D, Lewis KD, Ovcharenko I, Pachter L and Rubin EM: Phylogenetic shadowing of primate sequences to find functional regions of the human genome. Science 2003, 299(56 II):|39|-1394.

8. Grad Y, Aach J, Hayes GD, Reinhart BJ, Church GM, Ruvkun G and $\mathrm{Kim}$ J: Computational and experimental identification of Celegans microRNAs. Molecular Cell 2003, II(5):1253-1263.

9. Bentwich I, Avniel A, Karov Y, Aharonov R, Gilad S, Barad O, Barzilai $A$, Einat $P$, Einav $U$ and Meiri $E$, et al: Identification of hundreds of conserved and nonconserved human microRNAs. Nature Genetics 2005, 37(7):766-770.

10. Berezikov E, Guryev V, Belt van de J, Wienholds E, Plasterk RHA and Cuppen E: Phylogenetic shadowing and computational identification of human microRNA genes. Cell 2005, I 20(I):2I-24.

II. Sewer A, Paul N, Landgraf P, Aravin A, Pfeffer S, Brownstein MJ, Tuschl $T$, van Nimwegen $E$ and Zavolan $M$ : Identification of clustered microRNAs using an ab initio prediction method. BMC Bioinformatics 2005, 6 .

12. Xue CH, Li F, He T, Liu GP, Li YD and Zhang XG: Classification of real and pseudo microRNA precursors using local structuresequence features and support vector machine. BMC Bioinformatics 2005, 6 .

13. Brameier $M$ and Wiuf $C:$ Ab initio identification of human microRNAs based on structure motifs. BMC Bioinformatics 2007, 8.
14. Kwang Loong S and Mishra SK: De novo SVM classification of precursor microRNAs from genomic pseudo hairpins using global and intrinsic folding measures. Bioinformatics 2007, 23 (II): $1321-1330$.

15. Chang DTH, Wang CC and Chen JW: Using a kernel density estimation based classifier to predict species-specific microRNA precursors. BMC Bioinformatics 2008, 9.

16. Schultes EA, Hraber PT and LaBean TH: Estimating the contributions of selection and self-organization in RNA secondary structure. J Mol Evol 1999, 49(1):76-83.

17. Zhang BH, Pan XP, Cox SB, Cobb GP and Anderson TA: Evidence that miRNAs are different from other RNAs. Cell Mol Life Sci 2006, 63(2):246-254.

18. Freyhult E, Gardner PP and Moulton V: A comparison of RNA folding measures. BMC Bioinformatics 2005, 6.

19. Gan HH, Fera D, Zorn J, Shiffeldrim N, Tang M, Laserson U, Kim N and Schlick T: RAG: RNA-As-Graphs database - concepts, analysis, and features. Bioinformatics 2004, 20(8):|285-|29|.

20. Nam JW, Shin KR, Han J], Lee Y, Kim VN and Zhang BT: Human microRNA prediction through a probabilistic co-learning model of sequence and structure. Nucleic Acids Res 2005, 33 (I I):3570-358I.

21. Terai G, Komori T, Asai K and Kin T: miRRim: A novel system to find conserved miRNAs with high sensitivity and specificity. RNA-a Publication of the RNA Society 2007, I 3(12):208 I-2090.

22. Yang YC, Wang YP and $\mathrm{Li}$ KB: MiRTif: a support vector machine-based microRNA target interaction filter. $B M C$ Bioinformatics 2008, 9.

23. Batuwita $R$ and Palade $V$ : microPred: effective classification of pre-miRNAs for human miRNA gene prediction. Bioinformatics 2009, 25(8):989-995.

24. Jain AK, Duin RPW and Mao JC: Statistical pattern recognition: A review. IEEE Transactions on Pattern Analysis and Machine Intelligence 2000, 22(I):4-37.

25. Huang LT, Gromiha MM and Ho SY: iPTREE-STAB: interpretable decision tree based method for predicting protein stability changes upon mutations. Bioinformatics 2007, 23 (10): I 292-I 293 .

26. Zhou XF, Ruan JH, Wang GD and Zhang WX: Characterization and identification of microRNA core promoters in four model species. PLoS Comput Biol 2007, 3(3):4|2-423.

27. Ho SY, Hsieh CH, Chen HM and Huang HL: Interpretable gene expression classifier with an accurate and compact fuzzy rule base for microarray data analysis. Biosystems 2006, 85 (3): 165-176.

28. Zhou GD: Recognizing names in biomedical texts using mutual information independence model and SVM plus sigmoid. Int J Med Inf 2006, 75(6):456-467.

29. Hsieh C-H, Chang DT-H and Oyang Y-J: Data Classification with a Generalized Gaussian Components based Density Estimation Algorithm. International Joint Conference on Neural Networks. Atlanta, Georgia 2009.

30. Ritchie W, Legendre $M$ and Gautheret D: RNA stem-loops: To be or not to be cleaved by RNAse III. RNA 2007, I3(4):457-462.

31. Chang DTH, Oyang YJ and Lin JH: MEDock: a web server for efficient prediction of ligand binding sites based on a novel optimization algorithm. Nucleic Acids Res 2005, 33:W233-W238.

32. Oyang YJ, Hwang SC, Ou YY, Chen CY and Chen ZW: Data classification with radial basis function networks based on a novel kernel density estimation algorithm. IEEE Transactions on Neural Networks 2005, I 6(I):225-236.

33. Han LY, Cai CZ, Lo SL, Chung MCM and Chen YZ: Prediction of RNA-binding proteins from primary sequence by a support vector machine approach. RNA 2004, I 0(3):355-368.

34. Dror G, Sorek R and Shamir R: Accurate identification of alternatively spliced exons using support vector machine. Bioinformatics 2005, 21 (7):897-901.

35. Quinlan JR: C4.5: Programs for Machine Learning.San Francisco: Morgan Kaufmann; 1993.

36. Cohen WW: Fast effective rule induction. International Conference on Machine Learning 1995 1995, II5-123.

37. Wilcoxon F: Individual Comparisons by Ranking Methods. Biometrics Bulletin 1945, I (6):80-83.

38. Hogg RV and Tanis EA: Probability and statistical inference. Upper Saddle River, NJ: Pearson Prentice Hall; 72006.

39. Seffens $W$ and Digby $D$ : mRNAs have greater negative folding free energies than shuffled or codon choice randomized sequences. Nucleic Acids Res 1999, 27(7):I578-1584.

40. Moulton V, Zuker M, Steel M, Pointon R and Penny D: Metrics on RNA secondary structures. J Comput Biol 2000, 7(I-2):277-292. 
4I. Fera D, Kim N, Shiffeldrim N, Zorn J, Laserson U, Gan HH and Schlick T: RAG: RNA-As-Graphs web resource. BMC Bioinformatics 2004, 5

42. Larsen RJ and Marx ML: An Introduction to Mathematical Statistics and Its Applications.Prentice Hall; 32005.

43. Griffiths-Jones S, Grocock RJ, van Dongen S, Bateman A and Enright AJ: miRBase: microRNA sequences, targets and gene nomenclature. Nucleic Acids Res 2006, 34:DI40-D I 44.

44. Pruitt KD and Maglott DR: RefSeq and LocusLink: NCBI genecentered resources. Nucleic Acids Res 200 I, 29(I): I37-| 40.

45. Karolchik D, Baertsch R, Diekhans M, Furey TS, Hinrichs A, Lu YT, Roskin KM, Schwartz M, Sugnet CW and Thomas DJ, et al: The UCSC Genome Browser Database. Nucleic Acids Res 2003, 3 I (I):5 I-54.

46. Hofacker IL: Vienna RNA secondary structure server. Nucleic Acids Res 2003, 3 I (I3):3429-343 I.

47. Chang CC and Lin C): LIBSVM: a library for support vector machines. $200 \mathrm{I}$ http://www.csie.ntu.edu.tw/ cjlin/libsvm.

Publish with Bio Med Central and every scientist can read your work free of charge

"BioMed Central will be the most significant development for disseminating the results of biomedical research in our lifetime. "

Sir Paul Nurse, Cancer Research UK

Your research papers will be:

- available free of charge to the entire biomedical community

- peer reviewed and published immediately upon acceptance

- cited in PubMed and archived on PubMed Central

- yours - you keep the copyright
BioMedcentral 